\title{
Abacavir/Lamivudine Fixed-Dose Combination Antiretroviral Therapy for the Treatment of HIV
}

\author{
Chad J. Achenbach, Kimberly K. Scarsi, and Robert L. Murphy \\ Feinberg School of Medicine and Center for Global Health, Northwestern University, Chicago, \\ USA
}

Chad J. Achenbach: c-achenbach@northwestern.edu

\begin{abstract}
In the past 15 years, improvements in the treatment of HIV infection have dramatically reduced morbidity and mortality. Nucleoside reverse transcriptase inhibitors are the backbone of combination antiretroviral therapy for the treatment of HIV. One of the recommended and commonly used therapies in this class is the once-daily fixed-dose combination of abacavir/ lamivudine. Clinical studies and practice have shown these drugs to be potent, safe, and easy to use in a variety of settings; however, several recent reports have challenged the safety and efficacy claims among certain patient populations, including those at risk for cardiovascular disease and in those with high viral loads prior to treatment initiation. We reviewed abacavir/lamivudine as a treatment for HIV and discussed limitations of its use due to these controversial issues.
\end{abstract}

\section{Keywords}

abacavir; antiretroviral therapy; Epzicom; HIV; lamivudine; nucleoside reverse transcriptase inhibitor

\section{INTRODUCTION}

HIV is a major public health problem with an estimated 33 million infected adults worldwide, an estimated 2.7 million new infections per year, and 2 million deaths from the disease annually. ${ }^{1}$ Despite these dismal statistics, treatment of HIV infection has improved dramatically over the past 15 years due to the development of simple and effective combination antiretroviral therapy (cART). Incidence rates of AIDS-defining conditions and HIV-related mortality have decreased considerably with advances in cART. ${ }^{2,3}$ Over time, medications for the treatment of HIV have become simpler, less toxic, and more potent. There are now several effective cART regimens with a burden of one to three pills dosed once daily, which requires minimal clinical monitoring. These advancements have allowed cART to be rolled out extensively to primary care clinics in both resource-rich and -poor settings throughout the world. 
As of 2008, the International AIDS Society (IAS) and the British HIV Association (BHIVA) recommend two nucleoside reverse transcriptase inhibitors (NRTIs) plus either efavirenz (a non-nucleoside reverse transcriptase inhibitor [NNRTI]) or a protease inhibitor (PI) boosted with a low dose of ritonavir for initial cART of HIV-infected adults. ${ }^{4,5}$ Preferred NRTI components of cART are fixed-dose combinations (FDC) of tenofovir and emtricitabine or abacavir and lamivudine. More recently, the 2009 guidelines from the US Department of Health and Human Services (DHHS) ${ }^{6}$ have recommended tenofovir and emtricitabine or lamivudine as the preferred NRTIs in an initial cART regimen. For several reasons that will be discussed further in this review, abacavir is now considered an alternative to tenofovir by the DHHS. A 2009 update to World Health Organization (WHO) guidelines does not include abacavir in first-line cART. ${ }^{7}$ Finally, revisions to IAS and BHIVA guidelines in the near future are also expected to downgrade abacavir to an alternate agent.

An important development in the advancement of HIV treatment was the FDC of abacavir/ lamivudine (Epzicom and Kivexa; GlaxoSmithKline, Brentford, Middlesex, UK) which is FDA-approved for the treatment of HIV as a part of cART. It has been widely used throughout the US, Europe, and in some resource-limited settings due to its ease of dosing, favorable toxicity profile, and virologic potency. Despite extensive past use, recent controversial findings regarding abacavir/ lamivudine-FDC and one of its components, abacavir, may limit future use of this compound. In particular, patients on abacavir can develop a rare, but potentially life-threatening hypersensitivity reaction. ${ }^{8,9}$ Also, in several large, observational studies, abacavir has been associated with myocardial infarction (MI). ${ }^{10-13}$ Finally, in a recent randomized trial, abacavir/ lamivudine-FDC was found to be inferior to tenofovir/emtricitabine (Truvada; Gilead Sciences, Foster City, CA, USA) with regard to virologic suppression in patients with higher HIV viral load levels. ${ }^{14}$ We reviewed the treatment of HIV with abacavir/ lamivudine-FDC and these recent controversial findings that will likely limit its future use.

\section{MECHANISM OF ACTION AND PHARMACOLOGY}

Abacavir/lamivudine-FDC has demonstrated bioequivalence to the individual components of abacavir $600 \mathrm{mg}$ and lamivudine $300 \mathrm{mg}$ given once daily. ${ }^{8,15}$ This makes the combination product a preferable formulation due to ease of administration and lower pill burden, without jeopardizing any therapeutic effect. Pharmacologic properties of the combination tablet can be interpreted from the individual components as discussed below.

\section{Abacavir}

Abacavir is a synthetic carbocyclic nucleoside analog, which requires intracellular phosphorylation to its active anabolite, carbovir triphosphate (CBV-TP), similar to other NRTIs. CBV-TP inhibits transcription of HIV viral RNA to DNA by competing with an endogenous nucleotid*transcriptase enzyme. The nucleoside substitution of CBV-TP lacks the 3'hydroxyl group required for DNA polymerization, resulting in chain termination. Abacavir is a potent antiviral agent which can inhibit both HIV-1 and HIV-2, while demonstrating only weak inhibition of human cellular DNA polymerases $a, \beta$, and $\gamma^{8}$ 
Abacavir tablets are $83 \%$ bioavailable after oral administration and rapidly reach peak plasma concentrations between 0.63 to 2.5 hours. ${ }^{16,17}$ Abacavir is modestly affected by administration with food, both in time to maximum concentration $\left(\mathrm{T}_{\max }\right)$ and the maximum concentration achieved $\left(\mathrm{C}_{\max }\right)$. When administered with food, the $\mathrm{T}_{\max }$ is delayed (range 1.39-2.5 hours) compared to the fasted state (range $0.63-1.53$ hours), ${ }^{17}$ and the $\mathrm{C}_{\max }$ is decreased 26\% (90\% CI: 0.84, 0.65). ${ }^{16}$ Despite the changes observed when abacavir is given with food, the extent of drug exposure is unchanged, represented by the area under the curve (AUC). Therefore, abacavir can be given with or without food. ${ }^{18}$

Abacavir is well distributed throughout the body, with a volume of distribution of $0.86 \pm 0.15$ $\mathrm{L} / \mathrm{Kg}$, and is approximately $50 \%$ protein-bound. Despite this moderate protein binding, several reports have shown that the cerebrospinal fluid concentration of abacavir is only $27 \%-36 \%$ of the plasma concentration. ${ }^{17}$ Abacavir is extensively distributed across the placenta, demonstrated as a ratio of abacavir in cord blood to maternal plasma of $1.1 \pm 0.32$ at the time of delivery. ${ }^{19}$

Unlike most NRTIs, only $1 \%$ of abacavir is found unchanged in the urine after oral administration. The abacavir pharmacokinetics profile is not significantly impacted by renal insufficiency, therefore, dosage adjustment of abacavir is not required regardless of the patient's degree of renal dysfunction. ${ }^{20,21}$ This is an important consideration when selecting NRTIs for initial cART since the tenofovir component of tenofovir/emtricitabine-FDC requires renal adjustment and can cause further renal impairment. During a 4-hour highpermeability membrane dialysis session, the abacavir extraction ratio was $24 \%$. This suggests no supplemental dose of abacavir is required after hemodialysis, but the dose should be administered after the dialysis session to minimize drug clearance, particularly when abacavir is given as a once-daily dose. ${ }^{20}$ Abacavir is metabolized into two inactive metabolites via uridine diphosphate glucuronyl transferase and alcohol dehydrogenase. These inactive metabolites are then eliminated through the kidneys. ${ }^{16}$ In patients with mild hepatic impairment (Child-Pugh scores 5-6) the AUC was increased by $89 \%$ and the plasma elimination half-life $\left(\mathrm{T}_{1 / 2}\right)$ was $58 \%$ longer than in patients with normal hepatic function after a single dose of abacavir $600 \mathrm{mg}$. Therefore, a reduced dose of abacavir (200 mg twice daily) is recommended for patients with a Child-Pugh score of 5-6, and abacavir is currently not recommended for patients with Child-Pugh scores $>6 .{ }^{18}$ Given this required dose reduction, abacavir/ lamivudine-FDC should not be used in patients with hepatic insufficiency.

The plasma $\mathrm{T}_{1 / 2}$ of abacavir is estimated to be $1.45 \pm 0.32$ hours. ${ }^{8}$ Since the pharmacologically active moiety of abacavir is the intracellular CBV-TP, the pharmacokinetics of CBV-TP are essential for determining the appropriate dosing interval for abacavir. Intracellular CBV-TP has wide within-patient variability, making it difficult to estimate pharmacokinetic parameters. One study found CBV-TP had an average $\mathrm{T}_{1 / 2}$ of 14.1 hours, but the range was $4.8-39$ hours. $^{22}$ Other studies of intracellular CBV-TP elimination have also demonstrated a $\mathrm{T}_{1 / 2}$ mean greater than 12 hours, supporting once-daily dosing. ${ }^{23-25}$ When comparing abacavir $300 \mathrm{mg}$ twice daily to abacavir $600 \mathrm{mg}$ once-daily dosing, the once-daily dosing resulted in $32 \%$ higher CBV-TP AUC $0-24$ (90\% CI: 1.07 , 1.63). ${ }^{22}$ In studies evaluating a pharmacokinetic-pharmacodynamic relationship, the total 
exposure to abacavir (or AUC) is the pharmacokinetic parameter that has been associated with HIV-RNA decline and CD4+ cell count improvement. ${ }^{26}$ Therefore, these pharmacokinetic data support the non-inferiority clinical outcome data between the two regimens, ${ }^{27}$ and once-daily abacavir dosing is considered interchangeable with twice-daily dosing.

Abacavir is not a substrate for the cytochrome P450 enzyme system, minimizing its potential for drug interactions via this mechanism. Abacavir does not appear to have any adverse pharmacokinetic drug interactions with other NRTIs, including adefovir, tenofovir, lamivudine, and zidovudine. ${ }^{17,28,29}$ Despite this lack of pharmacokinetic interaction, there is evidence that the combination of abacavir, tenofovir, and lamivudine results in suboptimal virologic outcomes. It is suggested that this clinical observation may be related to a pharmacodynamic interaction resulting in selective pressure for the development of certain resistance mutations. ${ }^{30}$ Minor reductions in abacavir exposure have been observed when dosing with PI-based regimens including ritonavir (17\%-40\% decrease); however, no decrease in abacavir was observed with indinavir or amprenavir. ${ }^{31-33}$ No changes in the coadministered PI concentrations were observed in these studies. The proposed mechanism of decreased abacavir concentrations with ritonavir is induction of glucuronidation. As abacavir and alcohol share alcohol dehydrogenase as the primary metabolic pathway, the coadministration of these agents is of interest. When a single dose of abacavir $600 \mathrm{mg}$ was administered with the equivalent of approximately 4.7 glasses of wine, abacavir exposure increased $41 \%$ (90\% CI: 1.35, 1.48); however, there was no evidence of disulfuram-like reactions and no changes in alcohol concentrations. Since the increase in total abacavir exposure was within the therapeutic window observed in other studies, alcohol use is not contraindicated with abacavir. ${ }^{34}$ Finally, there is a potential interaction between abacavir and ribavirin that has been observed in clinical studies of patients undergoing hepatitis $\mathrm{C}$ virus treatment. Suboptimal response to hepatitis $\mathrm{C}$ treatment was associated with abacavir use, hypothesized to be related to impaired ribavirin intracellular phosphorylation due to competition between these two guanosine analogs. ${ }^{35}$ This potential interaction has not been properly evaluated in pharmacokinetic studies to date.

\section{Lamivudine}

Lamivudine is a synthetic nucleoside analog, which is a negative enantiomer of a cytidine analog. Similar to other NRTIs, lamivudine undergoes intracellular phosphorylation to its active anabolite, lamivudine-triphosphate (3TC-TP). Lamivudine shares the same mechanism of action against HIV as abacavir, resulting in DNA chain termination after incorporation of 3TC-TP in place of endogenous cytidine triphosphate by the reverse transcriptase enzyme. Lamivudine has potent antiviral effects on HIV-1, HIV-2, and hepatitis B viruses, but only weakly inhibits human cellular DNA polymerases $\alpha, \beta$, and $\gamma{ }^{8}$

Lamivudine is well absorbed via passive diffusion across the intestinal wall, with a reported bioavailability of $86 \%$ after oral administration and $\mathrm{C}_{\max }$ reached between 0.5 and 1.5 hours postdose. ${ }^{8,36}$ When given with food, the $\mathrm{C}_{\max }$ is reduced by 40 minutes $( \pm 23 \%)$ compared to fasted state, and the $\mathrm{T}_{\max }$ is delayed (3.2 \pm 1.3 hours in the fed state vs. $0.9 \pm 0.3$ hours under 
fasting conditions). Despite these changes, the total lamivudine exposure (the AUC) is unchanged, therefore lamivudine may be given irrespective of food. ${ }^{37}$

Lamivudine is not extensively protein bound $(<36 \%)$ and is well distributed throughout the body (volume of distribution $1.3 \pm 0.4 \mathrm{~L} / \mathrm{kg}$ ). ${ }^{37}$ Despite this low protein binding and broad distribution of drug, lamivudine is not extensively distributed into the cerebrospinal fluid (CSF), demonstrated by a mean CSF-to-serum ratio of $0.06 .{ }^{38}$ However, lamivudine is very well distributed from maternal to fetal circulation with maternal, cord, and neonate blood levels nearly equivalent at delivery 39

Similar to other NRTIs, renal elimination via filtration and active tubular secretion is the primary route of lamivudine clearance. Lamivudine clearance is correlated linearly with estimated creatinine clearance in patients with renal insufficiency, resulting in higher lamivudine concentrations and longer lamivudine $\mathrm{T}_{1 / 2}$ compared to patients with normal renal function. ${ }^{40}$ Therefore, dose reductions of lamivudine are recommended when the estimated creatinine clearance falls below $50 \mathrm{~mL}$. Due to this required dose reduction, abacavir/lamivudine-FDC cannot be used in patients with moderate to severe renal insufficiency. ${ }^{8}$ Only $5 \%-10 \%$ of lamivudine is metabolized to an inactive trans-sulfoxide metabolite, which is eliminated via the kidneys. Pharmacokinetic parameters were unchanged in an evaluation of a single dose of lamivudine in patients with hepatic insufficiency. ${ }^{41}$ Therefore, lamivudine does not require dosage adjustment in patients with hepatic impairment. ${ }^{37}$

Plasma $\mathrm{T}_{1 / 2}$ of lamivudine is estimated to be 5-7 hours. ${ }^{8,36,42}$ However, similar to abacavir, the intracellular pharmacokinetics of the pharmacologically active 3TC-TP are more relevant in determining the frequency of lamivudine dosing. Initial studies of lamivudine $150 \mathrm{mg}$ twice daily found an intracellular $\mathrm{T}_{1 / 2}$ of 15.3 (range 6.2-32) hours, suggesting a minimum dosing interval of 12 hours. ${ }^{43}$ Given this long intracellular half-life, subsequent studies compared the pharmacokinetic parameters of lamivudine given as $150 \mathrm{mg}$ twice daily versus $300 \mathrm{mg}$ once daily and found that the total exposure and $\mathrm{T}_{1 / 2}$ were similar for both the plasma lamivudine and intracellular 3TC-TP concentrations. Minimum concentrations were $18 \%$ to $24 \%$ lower in the once-daily dosing arms; however, the clinical significance of these reductions could not be evaluated. ${ }^{44,45}$ In a 48-week comparative study of once-daily versus twice-daily dosing of lamivudine in combination with zidovudine and efavirenz, the two dosing strategies demonstrated equivalence in HIV viral load response and CD4+ cell count changes. ${ }^{46}$ As a result, once-daily and twice-daily dosing of lamivudine are considered pharmacologically and clinically equivalent.

Lamivudine is not a substrate, inducer, or inhibitor of the cytochrome P450 enzyme system, minimizing the potential for drug interactions. Several HIV NRTI agents have been investigated in combination with lamivudine resulting in no pharmacokinetic interactions, including tenofovir, abacavir, zidovudine, and didanosine. ${ }^{23,38,47,48}$ Based on in-vitro data, antagonism between lamivudine and ribavirin was thought to exist; however, subsequent invivo data suggest that there is no interaction. ${ }^{49}$ Coadministration of trimethoprim and lamivudine results in a $43 \%(90 \% \mathrm{CI}: 1.32,1.55)$ increase in the lamivudine AUC due to competition between lamivudine and trimethoprim for the same renal transport system. ${ }^{42}$ 
Despite this observed increase in lamivudine concentrations, coadministration of these agents is not contraindicated.

\section{SAFETY AND TOLERABILITY}

In antiretroviral-naïve patients, the safety and tolerability of once-daily abacavir/ lamivudine-FDC has been extensively studied in comparison to twice-daily dosing of abacavir and lamivudine. ${ }^{50,51}$ Overall, the frequency and type of adverse events are similar whether or not the drugs are dosed once daily as a FDC or twice daily. In general, abacavir/ lamivudine-FDC is considered to be well tolerated with the most common ( $25 \%$ ) moderate to severe adverse events of insomnia, depression, headache, fatigue, dizziness, diarrhea, and rash. ${ }^{8}$ In some studies, a difference in abacavir hypersensitivity between once and twicedaily dosing was observed, which will be discussed further below.

\section{Abacavir Hypersensitivity}

The most common and concerning moderate to severe short-term adverse reaction related to abacavir/lamivudine-FDC is hypersensitivity to abacavir. Possible or suspected abacavir hypersensitivity reactions have been reported in $2 \%-10 \%$ of patients on this therapy. ${ }^{9,14,27,52-54}$ Abacavir hypersensitivity is a multiorgan clinical syndrome with $95 \%$ of effected patients experiencing at least two of the following groups of symptoms: fever, rash, gastrointestinal (nausea, vomiting, diarrhea, or abdominal pain), constitutional (generalized malaise, fatigue, or achiness), and respiratory (dyspnea, cough, or pharyngitis). ${ }^{8}$ This reaction can occur at any time with a median time to onset of 9 days, while $90 \%$ develop symptoms within the first 6 weeks of starting abacavir.

Several studies have examined differences in hypersensitivity reactions between abacavir dosed once daily versus twice daily. ${ }^{27,50,51}$ Initially, concern was raised after one blinded randomized trial showed a nonsignificant difference of severe hypersensitivity reaction for once-versus twice-daily abacavir dosing (5\% vs. $2 \%$, respectively, $P=0.28$ ) ${ }^{47}$ Subsequently, a larger randomized clinical trial found the opposite result with less severe hypersensitivity among those on once- compared to twice-daily dosing ( $0.9 \%$ vs. $2.2 \%$, respectively). ${ }^{50} \mathrm{In}$ addition, there were no hypersensitivity reactions in either arm of a study where 260 patients were randomized to switch from twice-daily abacavir and lamivudine to abacavir/ lamivudine-FDC. ${ }^{54}$ Finally, a large retrospective analysis of data compiled on abacavir hypersensitivity reactions from 37 clinical trials with 9330 patients found no difference in severe reactions among those on once-daily compared to twice-daily abacavir dosing. ${ }^{55}$

Currently, concern over abacavir hypersensitivity with the use of abacavir/lamivudine-FDC has largely abated as HLA-B*5701 testing has become common practice. Several years ago, two independent research groups discovered that there was a strong association between carriage of the HLA-B*5701 allele and this drug reaction. ${ }^{56,57}$ Since then, several prospective studies in different parts of the world have confirmed these findings. The strongest of these studies was the Prospective Randomized Evaluation of DNA Screening in a Clinical Trial (PREDICT-1). ${ }^{58}$ At 265 sites in 19 countries, 1956 patients naïve to abacavir were randomized to pharmacogenetic testing for HLA-B*5701 (study arm) versus no HLA testing (control arm) prior to starting cART containing abacavir. In the study arm, patients 
received an alternative NRTI in place of abacavir if they tested HLA positive. All the control patients received HLA testing at the end of the study. Overall, the carriage of HLA$\mathrm{B} * 5701$ allele was $5.6 \%$ for both arms and testing for the allele led to a significant reduction in the incidence of clinical and immunologic abacavir hypersensitivity. The incidence of clinical hypersensitivity was $7.8 \%$ in the control arm compared to $3.4 \%$ in the HLA screened arm $(P<0.001)$. Even more convincing was the difference in immunologic abacavir sensitivity of $2.7 \%$ in the control arm compared to no cases in the screened arm $(P<0.001)$ as measured by abacavir epi-cutaneous patch testing. In addition, among those with clinical reactions, but negative immunologic testing, subgroup analyses found significant associations with starting a new NNRTI or PI at the same time as abacavir. This suggested another drug reaction was a potential cause of the reactions attributed to abacavir hypersensitivity. The only variable significantly associated with immunologically confirmed abacavir hypersensitivity was carriage of the HLA-B*5701 allele. In summary, in this large multi-center randomized clinical trial, screening patients of largely European origin for HLA-B*5701 effectively excluded all cases of immunologically confirmed abacavir hypersensitivity.

As a result of all these findings, HLA-B*5701 screening of all patients prior to treatment with abacavir is recommended in international HIV treatment guidelines. ${ }^{5}$ As approximately $50 \%$ of HLA-B*5701-positive patients develop immunologically confirmed abacavir hypersensitivity reactions, they should not receive abacavir. A positive HLA-B*5701 test should be clearly documented in the medical record and the patient should be labeled as having an allergy to all abacavir-containing products. In addition, negative HLA testing should not be used as a replacement for careful clinical follow-up and monitoring for these potentially serious reactions among patients on abacavir.

\section{Abacavir and MI}

One of the most controversial issues in HIV treatment over the past few years has been whether or not there is an association between treatment with abacavir and MI. The controversy began when analyses of the Data Collection on Adverse Events of Anti-HIV Drugs (D:A:D) study, which included over 30,000 HIV-infected individuals in 11 cohorts, revealed an increased risk of MI among patients exposed to abacavir and didanosine, another NRTI agent. ${ }^{10}$ After adjustments for demographics, cardiovascular disease history, smoking, and exposure to other anti-retroviral drugs, recent (but not cumulative) exposure to abacavir was independently associated with MI (relative risk [RR] 1.90; 95\% CI: 1.47, 2.45; $P<0.01)$. The increase in MI rate was most notable among those with a high predicted 10year risk of coronary heart disease (CHD) (Figure 1). ${ }^{10}$ Analyses were recently repeated with additional follow-up time and more patients on other NRTIs, including tenofovir, with similar results (adjusted RR 1.70; 95\% CI: 1.17, 2.47)..$^{59}$

These findings were unanticipated since abacavir has not been shown to adversely affect lipids, glucose, insulin, or insulin resistance. ${ }^{53,60-62}$ In fact, early in its development and use, abacavir was often prescribed to patients thought to be at risk for CHD. This predisposition was shown in the D:A:D study where $27 \%$ of patients starting abacavir had moderate or high predicted 10-year risk of CHD compared to $19 \%$ of those starting other 
NRTIs such as zidovudine or stavudine. Critics of D:A:D have suggested this practice may have influenced the study through a channeling bias.

An analysis of a separate independent cohort of patients enrolled in one arm of the Strategies for Management of Anti-Retroviral Therapy (SMART) study confirmed these findings when they also noted an increased risk of clinical MI for those currently receiving abacavir (adjusted HR [hazard ratio] 4.25; 95\% CI: 1.41, 12.6; $P<0.05$ ). ${ }^{11}$ This study was considerably smaller with only 19 clinical MI events, and there was less disparity in cardiovascular risk profile for patients on different types of NRTIs compared to the D:A:D study. Five or more CHD risk factors were reported in 18\% of those on abacavir versus $14 \%$ of those on other NRTIs, suggesting channeling bias was less likely to have influenced this association.

In response to these two studies, the manufacturer of abacavir, GlaxoSmithKline, assembled safety data from over 14,000 adult patients enrolled in 52 clinical trials. ${ }^{63}$ Overall, MI rates were very low and there was no difference when comparing those with and without exposure to abacavir (2.09 vs. 2.57 per 1000 person-years, respectively; RR 0.81 ; $95 \%$ CI: $0.38,1.75)$. To minimize the potential for channeling bias, analyses were focused on 12 studies in which patients were randomly assigned to receive abacavir. Again, they reported similar incidence rates of MI for those with and without exposure to abacavir ( $2.1 \mathrm{vs.} 4.1$ per 1000 person-years, respectively; RR 0.52 ; $95 \%$ CI: $0.2,1.8$ ). Despite their analysis of randomized patients, a major criticism of this study was the lack of baseline information on all cardiovascular risk factors and inability to make adjustments for these important confounding variables. The AIDS Clinical Trials Group performed a similar pooled analysis of 3205 patients starting their first ART regimen, of which 781 were randomly assigned to abacavir, and they also found no association between recent abacavir exposure and MI (adjusted RR 1.2; 95\% CI: 0.5, 3.1, $P=0.82$ ). ${ }^{64}$

More evidence of an association between MI and abacavir use were reported from retrospective analyses of French and Danish HIV treatment databases. ${ }^{12,13}$ In the French study, after adjustment for cardiovascular risk factors, they originally found an increased risk in MI among those with less than 1 year of exposure, or recent use, of abacavir (odds ratio [OR] 2.2; 95\% CI: 1.2, 4.0); ${ }^{12}$ however, with further analysis, the association was found only in patients with active drug use. At a recent HIV conference in South Africa, presentation of two more retrospective analyses offered conflicting results. One from Quebec's public health insurance database again identified an association of MI with abacavir (OR 1.74; 95\% CI: 1.18, 2.56). ${ }^{65}$ The other from the US Veterans Administration Database reported that exposure to abacavir slightly increased the risk for MI, but the result was not statistically significant. ${ }^{66}$ In addition, any observed association was diminished when they controlled for cardiovascular risk factors, especially chronic kidney dysfunction defined by an estimated glomerular filtration of less than $60 \mathrm{~mL} / \mathrm{min}$. These findings again suggested channeling bias might be an issue as patients with renal failure, a known risk factor for cardiovascular disease, may have been preferentially prescribed abacavir instead of tenofovir. Supporting this possibility, a group from the UK found that among 1491 patients who received cART, 58\% with chronic kidney disease received abacavir compared to $22 \%$ of those without renal dysfunction $(P<0.001) .67$ 
Despite the epidemiologic associations, no study has demonstrated a clear biological mechanism to explain how abacavir alters the pathogenesis of cardiovascular disease and increases risk for MI. Analysis of patients in the SMART study revealed a greater proportion of patients on abacavir with elevated inflammatory markers of highly sensitive CRP (hsCRP) and interleukin-6, ${ }^{11}$ but several other studies have reported no differences in these measurements. ${ }^{53,68,69}$ Two small studies found significantly reduced endothelial function ${ }^{70}$ and increased platelet reactivity ${ }^{71}$ in patients treated with abacavir, but it is unknown how the mechanism or properties of abacavir may have led to changes in these biological parameters. Most uncertain is whether or not the use of abacavir is simply a marker of another underlying biological factor leading to alterations in cardiovascular pathophysiology.

In summary, several large clinical studies have conflicting results over the association between abacavir and MI. Once all confounding factors and channeling biases are controlled, it is uncertain how much of this association remains. Since there is not a current plausible biological mechanistic link between abacavir and MI, we do not know if these findings represent an association between abacavir and an undiscovered confounding risk factor. Therefore, at the current time, this drug appears to be safe for use in younger patients with minimal cardiovascular risk. For all HIV-infected patients on abacavir/lamivudineFDC, we should be vigilant about prevention, monitoring, and reduction of adverse cardiovascular risk factors.

\section{ABACAVIR/LAMIVUDINE-FDC EFFICACY}

As separate formulations, abacavir and lamivudine have been shown to have efficacy as the NRTI backbone of triple and quadruple cART for treatment of ART-naïve HIV-infected adults. In large randomized clinical trials, modern cART regimens containing these drugs dropped plasma HIV viral load levels to less than 50 copies $/ \mathrm{mL}$ after 48 weeks of therapy in approximately $70 \%$ of patients. ${ }^{27,72}$ In this same timeframe, cART containing these medications leads to an average increase in CD4+ T cell count of about 200 cells $/ \mathrm{mm}^{3}$.

Coformulation of abacavir and lamivudine into the FDC has similar virologic and immunologic efficacy to the products dosed separately. ${ }^{50,73}$ Simplification of cART regimens from twice-daily NRTIs to once-daily FDC has also proven to be effective at maintaining virologic suppression and immunologic recovery (Figure 2) ${ }^{52,54}$ In one of these studies, a larger proportion of subjects in the once-daily abacavir/lamivudine group (67\%) achieved greater than $95 \%$ adherence compared to those on twice-daily abacavir and lamivudine (53\%). ${ }^{73}$ As expected, overall patient satisfaction and adherence with the oncedaily FDC is excellent and comparable to or better than twice-daily dosing. ${ }^{50,54,73}$

Over the past few years, there has been great interest in determining the most effective dual NRTI backbone for initial cART. Specifically, recent clinical trials have compared efficacy and safety of the once-daily FDCs of abacavir/lamivudine and tenofovir/ emtricitabine. ${ }^{14,52,53}$ For simplification of dosing, one randomized clinical trial substituted abacavir/lamivudine-FDC with tenofovir/emtricitabine for twice-daily NRTIs in patients with virologic suppression on cART. ${ }^{52}$ Abacavir/lamivudine-FDC did not meet the non- 
inferiority outcome for treatment failure, defined as virologic rebound or switching cART, when compared to tenofovir/emtricitabine; however, a greater proportion of patients on abacavir/ lamivudine-FDC experienced treatment failure ( $19 \%$ vs. $13 \%, P=0.06)$. The study was performed at a time before HLA-B $* 5701$ screening and the higher treatment failure rate was driven by discontinuation of abacavir/lamivudine-FDC due to suspected abacavir hypersensitivity reactions.

Two large, randomized clinical trials compared abacavir/lamivudine-FDC and tenofovir/ emtricitabine as the NRTI backbone in initial cART among HIV-infected patients naïve to cART. ${ }^{14,53}$ One placebo-controlled and blinded study found no difference in HIV viral load suppression to less than 50 copies/mL for abacavir/ lamivudine-FDC compared to tenofovir/ emtricitabine-FDC, both given with lopinavir/ ritonavir, at either 48 (68\% vs. 67\%, respectively) or 96 (60\% vs. 58\%, respectively) weeks of treat-ment. ${ }^{53}$ In this study published by Smith et al., abacavir/lamivudine-FDC was virologically non-inferior to tenofovir/emtricitabine-FDC, regardless of baseline HIV viral load level (Figure 3). ${ }^{53}$ There were also no statistically significant differences in adverse events between the two FDCs. Suspected abacavir hypersensitivity was relatively uncommon ( $4 \%$ vs. $<1 \%$ for abacavir/ lamivudine-FDC and tenofovir/emtricitabine-FDC, respectively).

Despite these findings, there have been questions about the virologic efficacy of abacavir/ lamivudine-FDC for patients with baseline HIV viral load levels greater than 100,000 copies/mL. The ACTG 5202 study comparing abacavir/ lamivudine-FDC to tenofovir/ emtricitabine-FDC in initial cART, combined with efavirenz or atazanavir/ritonavir, was unblinded early after a data safety monitoring board noted a shorter time to virologic failure among patients on abacavir/lamivudine-FDC with baseline viral load greater than 100,000 copies $/ \mathrm{mL}$. The final analysis, recently published by Sax et al., ${ }^{14}$ reported virologic failure in $57(14 \%)$ patients in the abacavir/lamivudine-FDC group compared to $26(7 \%)$ patients in the tenofovir/ emtricitabine-FDC group. Virologic failure was defined as HIV viral load level $\geq 1000$ copies/ $\mathrm{mL}$ at or after 16 weeks and before 24 weeks, or $\geq 200$ copies $/ \mathrm{mL}$ at or after 24 weeks. They also noted the abacavir/lamivudine-FDC group had significantly shorter time to virologic failure than the tenofovir/emtricitabine-FDC group (adjusted HR 2.08; 95\% CI: 1.28, 3.37; $P<0.001$ ) (Figure 4). ${ }^{14}$ This difference in virologic outcome was found throughout the entire duration of the study and after they performed several sensitivity analyses. They found equal numbers of suspected hypersensitivity reactions between the two groups and this did not appear to influence the virologic results. Potential explanations were that abacavir/ lamivudine-FDC was less potent, had unfavorable pharmacokinetics, or expressed an adverse profile of emerging drug-resistant mutations. Currently, it is unknown exactly why patients treated with abacavir/lamivudine-FDC had a significantly higher virologic failure rate.

Important differences between this study and the study by Smith et al. ${ }^{53}$ include sample size, patient population, virologic endpoint definitions, third drug used in the cART regimen, and discontinuation rate. It is uncertain how these differences might have influenced the disparate virologic results between the two studies. 


\section{CONCLUSION}

For many years, abacavir/lamivudine-FDC has been successfully used in thousands of HIVinfected patients as the NRTI backbone of cART with minimal side effects and excellent virologic suppression. However, for several reasons, we suspect its use in initial cART for the treatment of HIV will decrease and be limited to certain patient populations. To minimize abacavir hypersensitivity reactions, HLA-B*5701 testing is recommended prior to starting abacavir/ lamivudine-FDC, which limits optimal use of the drug to those who can access and financially afford this screening test. Also, potential associations of abacavir with MI and abacavir/ lamivudine-FDC with virologic failure at higher viral loads limits the drug to those who are younger, have minimal cardiovascular risk, and lower baseline viral load levels. Finally, since abacavir/lamivudine-FDC is a fixed-dose combination medication, its dose cannot be reduced in the setting of hepatic insufficiency (for abacavir) or moderate to severe renal insufficiency (for lamivudine). Despite all these limitations, abacavir/ lamivudine-FDC will have a smaller, but important role in the management of HIV infection.

\section{ACKNOWLEDGMENTS}

None of the authors who contributed to this review have any personal, commercial, academic, or financial conflicts of interest except R.M., who has been a consultant to both Gilead Pharmaceuticals and GlaxoSmith Kline Pharmaceuticals.

\section{REFERENCES}

1. Report on the Global AIDS Epidemic 2008. [Last accessed February 11, 2010] UNAIDS web site. Available at: www.unaids.org/en/KnowledgeCentre/HIVData/GlobalReport/ 2008/2008_Global_report.asp

2. Palella FJ Jr, Delaney KM, Moorman AC, et al. Declining morbidity and mortality among patients with advanced human immunodeficiency virus infection. HIV Outpatient Study Investigators. N Engl J Med. 1998; 338:853-860. [PubMed: 9516219]

3. Mocroft A, Ledergerber B, Katlama C, et al. Decline in the AIDS and death rates in the EuroSIDA study: an observational study. Lancet. 2003; 362:22-29. [PubMed: 12853195]

4. Gazzard BG, Anderson J, Babiker A, et al. British HIV Association guidelines for the treatment of HIV-1-infected adults with antiretroviral therapy 2008. HIV Med. 2008; 9:563-608. [PubMed: 18826546]

5. Hammer SM, Eron JJ Jr, Reiss P, et al. Antiretroviral treatment of adult HIV infection: 2008 recommendations of the International AIDS Society-USA panel. JAMA. 2008; 300:555-570. [PubMed: 18677028]

6. Panel on Antiretroviral Guidelines for Adults and Adolescents. [Last accessed: February 11, 2010] Guidelines for the use of antiretroviral agents in HIV-1-infected adults and adolescents; Department of Health and Human Services. 2009 Dec 1. p. 1-161.Available at http://www.aidsinfo.nih.gov/ ContentFiles/AdultandAdo-lescentGL.pdf

7. [Last accessed: February 11, 2010] Rapid advice: antiretroviral therapy for HIV infection in adults and adolescents. World Health Organization ART Guideline Review Committee. 2009 Nov. Available at: www.who.int/hiv/pub/arv/rapid_advice_art.pdf

8. Epzicom (package insert). Research Triangle Park, NC: GlaxoSmithKline; 2009.

9. Nolan D. HLA-B*5701 screening prior to abacavir prescription: clinical and laboratory aspects. Crit Rev Clin Lab Sci. 2009; 46:153-165. [PubMed: 19514905] 
10. Sabin CA, Worm SW, Weber R, et al. Use of nucleoside reverse transcriptase inhibitors and risk of myocardial infarction in HIV-infected patients enrolled in the D:A:D study: a multi-cohort collaboration. Lancet. 2008; 371:1417-1426. [PubMed: 18387667]

11. Lundgren JD, Neuhaus J, Babiker A, et al. Use of nucleoside reverse transcriptase inhibitors and risk of myocardial infarction in HIV-infected patients. AIDS. 2008; 22:F17-F24. [PubMed: 18753925]

12. Lang, SM-KM.; Cotte, L.; Gilquin, L., et al. Impact of specific NRTI and PI exposure on the risk of myocardial infarction: a case-control study nested within FHDH ANRS C04; presented at: 16th Conference on Retroviruses and Oppotunistic Infections; February 8-11, 200; Montreal, Canada. p. 8-11.Abstract Abstract \#43LB

13. Obel N, Farkas D, Kronborg G, et al. Abacavir and risk of myocardial infarction in HIV-infected patients on highly active antiretroviral therapy: a population-based nationwide cohort study. HIV Med. 2010; 11:130-136. [PubMed: 19682101]

14. Sax PE, Tierney C, Collier AC, et al. Abacavir-lamivudine versus tenofovir-emtricitabine for initial HIV-1 therapy. N Engl J Med. 2009; 361:2230-2240. [PubMed: 19952143]

15. Baker, KL.; Lou, Y.; Yuen, G.; Murray, S.; Stein, D. The bioequivalence and effect of food on a new once-a-day fixed-dose combination tablet of abacavir (ABC) and lamivudine (3TC); Poster presented at: 44th Interscience Conference on Antimicrobial Agents and Chemotherapy; October 30, 2004 - November 2, 2004; Washington, DC.

16. Chittick GE, Gillotin C, McDowell JA, et al. Abacavir: absolute bioavailability, bioequivalence of three oral formulations, and effect of food. Pharmacotherapy. 1999; 19:932-942. [PubMed: 10453964]

17. Yuen GJ, Weller S, Pakes GE. A review of the pharmacokinetics of abacavir. Clin Pharmacokinet. 2008; 47:351-371. [PubMed: 18479171]

18. Ziagen (package insert). Research Park Triangle, NC: GlaxoSmithKline; 2009.

19. Best BM, Mirochnick M, Capparelli EV, et al. Impact of pregnancy on abacavir pharmacokinetics. AIDS. 2006; 20:553-560. [PubMed: 16470119]

20. Izzedine H, Launay-Vacher V, Aymard G, Legrand M, Deray G. Pharmacokinetics of abacavir in HIV-1-infected patients with impaired renal function. Nephron. 2001; 89:62-67. [PubMed: 11528234]

21. Foster RH, Faulds D. Abacavir. Drugs. 1998; 55:729-736. discussion 737-738. [PubMed: 9585869]

22. Moyle G, Boffito M, Fletcher C, et al. Steady-state pharmacokinetics of abacavir in plasma and intracellular carbovir triphosphate following administration of abacavir at 600 milligrams once daily and 300 milligrams twice daily in human immunodeficiency virus-infected subjects. Antimicrob Agents Chemother. 2009; 53:1532-1538. [PubMed: 19188387]

23. Hawkins T, Veikley W, St. Claire RL 3rd, Guyer B, Clark N, Kearney BP. Intracellular pharmacokinetics of tenofovir diphosphate, carbovir triphos-phate, and lamivudine triphosphate in patients receiving triple-nucleoside regimens. J Acquir Immune Defic Syndr. 2005; 39:406-411. [PubMed: 16010161]

24. Harris M, Back D, Kewn S, Jutha S, Marina R, Montaner JS. Intracellular carbovir triphosphate levels in patients taking abacavir once a day. AIDS. 2002; 16:1196-1197. [PubMed: 12004286]

25. Piliero, P.; Shachoy-Clark, AD.; Para, M., et al. A study examining the pharmacokinetics of abacavir and the intracellular carbovir triphosphate (GSK Protocol CNA 10905); presented at: 43rd International Conference on Antimicrobial Agents and Chemotherapy; September 14-17, 2003; Chicago, IL, USA. Abstract Abstract A-1797

26. Weller S, Radomski KM, Lou Y, Stein DS. Population pharmacokinetics and pharmacodynamic modeling of abacavir (1592U89) from a dose-ranging, double-blind, randomized monotherapy trial with human immunodeficiency virus-infected subjects. Antimicrob Agents Chemother. 2000; 44:2052-2060. [PubMed: 10898675]

27. Moyle GJ, DeJesus E, Cahn P, et al. Abacavir once or twice daily combined with once-daily lamivudine and efavirenz for the treatment of antiretroviral-naive HIV-infected adults: results of the Ziagen Once Daily in Antiretroviral Combination Study. J Acquir Immune Defic Syndr. 2005; 38:417-425. [PubMed: 15764958] 
28. Goicoechea, M.; Jain, S.; Kemper, C., et al., editors. Viral Dynamics and Pharmacokinetics in vivo of Tenofovir Disoproxil Fumarate and Abacavir: Evidence of a Non-additive Antiviral Effect; Poster presented at: 16th Conference on Retroviruses and Opportunistic Infections; February 8-11, 2009; Montréal, Canada. Poster no. 703. Available at: www.hivandhepatitis.com/2009icr/croi/pdf/ 3Golcoechea.pdf

29. McDowell JA, Lou Y, Symonds WS, Stein DS. Multiple-dose pharmacokinetics and pharmacodynamics of abacavir alone and in combination with zidovudine in human immunodeficiency virus-infected adults. Antimicrob Agents Chemother. 2000; 44:2061-2067. [PubMed: 10898676]

30. Mastroianni CM, d'Ettorre G, Vullo V. Evolving simplified treatment strategies for HIV infection: the role of a single-class quadruple-nucleoside/ nucleotide regimen of trizivir and tenofovir. Expert Opin Pharmacother. 2006; 7:2233-2241. [PubMed: 17059380]

31. DiCenzo R, Forrest A, Squires KE, et al. Indinavir, efavirenz, and abacavir pharmacokinetics in human immunodeficiency virus-infected subjects. Antimicrob Agents Chemother. 2003; 47:19291935. [PubMed: 12760869]

32. Waters L, Moyle G, D'Avolio A, et al. Abacavir plasma pharmacokinetics in the absence and presence of atazanavir/ritonavir or lopinavir/ ritonavir and vice versa in HIV-infected patients. Antivir Ther. 2007; 12:825-380. [PubMed: 17713166]

33. Aptivus (package insert). Ridgefield, CT: Boehringer Ingelheim Pharmaceuticals, Inc; 2009.

34. McDowell JA, Chittick GE, Stevens CP, Edwards KD, Stein DS. Pharmacokinetic interaction of abacavir (1592U89) and ethanol in human immunodeficiency virus-infected adults. Antimicrob Agents Chemother. 2000; 44:1686-1690. [PubMed: 10817729]

35. Bani-Sadr F, Denoeud L, Morand P, et al. Early virologic failure in HIV-coinfected hepatitis C patients treated with the peginterferon-ribavirin combination: does abacavir play a role? J Acquir Immune Defic Syndr. 2007; 45:123-125. [PubMed: 17460476]

36. Yuen GJ, Morris DM, Mydlow PK, Haidar S, Hall ST, Hussey EK. Pharmacokinetics, absolute bioavailability, and absorption characteristics of lamivudine. J Clin Pharmacol. 1995; 35:11741180. [PubMed: 8750368]

37. Epivir (package insert). Research Triangle Park, NC: GlaxoSmithKline; 2009.

38. Johnson MA, Moore KH, Yuen GJ, Bye A, Pakes GE. Clinical pharmacokinetics of lamivudine. Clin Pharmacokinet. 1999; 36:41-66. [PubMed: 9989342]

39. Moodley J, Moodley D, Pillay K, et al. Pharmacokinetics and antiretroviral activity of lamivudine alone or when coadministered with zidovudine in human immunodeficiency virus type 1-infected pregnant women and their offspring. J Infect Dis. 1998; 178:1327-1333. [PubMed: 9780252]

40. Heald AE, Hsyu PH, Yuen GJ, Robinson P, Mydlow P, Bartlett JA. Pharmacokinetics of lamivudine in human immunodeficiency virus-infected patients with renal dysfunction. Antimicrob Agents Chemother. 1996; 40:1514-1519. [PubMed: 8726029]

41. Johnson MA, Horak J, Breuel P. The pharmacokinetics of lamivudine in patients with impaired hepatic function. Eur J Clin Pharmacol. 1998; 54:363-366. [PubMed: 9696966]

42. Moore KH, Yuen GJ, Raasch RH, et al. Pharmacokinetics of lamivudine administered alone and with trimethoprim-sulfamethoxazole. Clin Pharmacol Ther. 1996; 59:550-558. [PubMed: 8646826]

43. Moore KH, Barrett JE, Shaw S, et al. The pharmacokinetics of lamivudine phosphorylation in peripheral blood mononuclear cells from patients infected with HIV-1. AIDS. 1999; 13:22392250. [PubMed: 10563709]

44. Bruno R, Regazzi MB, Ciappina V, et al. Comparison of the plasma pharmacokinetics of lamivudine during twice and once daily administration in patients with HIV. Clin Pharmacokinet. 2001; 40:695-700. [PubMed: 11605717]

45. Yuen GJ, Lou Y, Bumgarner NF, et al. Equivalent steady-state pharmacokinetics of lamivudine in plasma and lamivudine triphosphate within cells following administration of lamivudine at 300 milligrams once daily and 150 milligrams twice daily. Antimicrob Agents Chemother. 2004; 48:176-182. [PubMed: 14693537]

46. DeJesus E, McCarty D, Farthing CF, et al. Once-daily versus twice-daily lamivudine, in combination with zidovudine and efavirenz, for the treatment of antiretroviral-naive adults with 
HIV infection: a randomized equivalence trial. Clin Infect Dis. 2004; 39:411-418. [PubMed: 15307010]

47. Pruvost A, Negredo E, Theodoro F, et al. Pilot pharmacokinetic study of human immunodeficiency virus-infected patients receiving tenofovir disoproxil fumarate (TDF): investigation of systemic and intracellular interactions between TDF and abacavir, lamivudine, or lopinavir-ritonavir. Antimicrob Agents Chemother. 2009; 53:1937-1943.

48. Yuen GJ, Lou Y, Thompson NF, et al. Abacavir/ lamivudine/zidovudine as a combined formulation tablet: bioequivalence compared with each component administered concurrently and the effect of food on absorption. J Clin Pharmacol. 2001; 41:277-288. [PubMed: 11269568]

49. Rodriguez-Torres M, Torriani FJ, Soriano V, et al. Effect of ribavirin on intracellular and plasma pharmacokinetics of nucleoside reverse transcriptase inhibitors in patients with human immunodeficiency virus-hepatitis $\mathrm{C}$ virus coinfection: results of a randomized clinical study. Antimicrob Agents Chemother. 2005; 49:3997-4008. [PubMed: 16189072]

50. Cohen CJ, Kubota M, Brachman PS, et al. Short-term safety and tolerability of a once-daily fixeddose abacavir-lamivudine combination versus twice-daily dosing of abacavir and lamivudine as separate components: findings from the ALOHA study. Pharmacotherapy. 2008; 28:314-322. [PubMed: 18294111]

51. Goedken AM, Herman RA. Once-daily abacavir in place of twice-daily administration. Ann Pharmacother. 2005; 39:1302-1308. [PubMed: 15956231]

52. Martinez E, Arranz JA, Podzamczer D, et al. A simplification trial switching from nucleoside reverse transcriptase inhibitors to once-daily fixed-dose abacavir/lamivudine or tenofovir/ emtricitabine in HIV-1-infected patients with virological suppression. J Acquir Immune Defic Syndr. 2009; 51:290-297. [PubMed: 19398921]

53. Smith KY, Patel P, Fine D, et al. Randomized, double-blind, placebo-matched, multicenter trial of abacavir/lamivudine or tenofovir/emtricitabine with lopinavir/ritonavir for initial HIV treatment. AIDS. 2009; 23:1547-1556. [PubMed: 19542866]

54. Sosa N, Hill-Zabala C, Dejesus E, et al. Abacavir and lamivudine fixed-dose combination tablet once daily compared with abacavir and lamivudine twice daily in HIV-infected patients over 48 weeks (ESS30008, SEAL). J Acquir Immune Defic Syndr. 2005; 40:422-427. [PubMed: 16280696]

55. Brothers, CCA.; Zhao, H.; Edwards, M.; Fleming, J.; Scott, T. Once daily administration of abacavir is not a clinical risk factor for suspected hypersensitivity reactions in clinical trials and rash is not sufficient to diagnose the reaction; Poster presented at: 12th Conference on Retroviruses and Opportunistic Infections; February 23, 2005; Boston, USA. Poster 836

56. Mallal S, Nolan D, Witt C, et al. Association between presence of HLA-B*5701, HLA-DR7, and HLA-DQ3 and hypersensitivity to HIV-1 reverse-transcriptase inhibitor abacavir. Lancet. 2002; 359:727-732. [PubMed: 11888582]

57. Hetherington S, Hughes AR, Mosteller M, et al. Genetic variations in HLA-B region and hypersensitivity reactions to abacavir. Lancet. 2002; 359:1121-1122. [PubMed: 11943262]

58. Mallal S, Phillips E, Carosi G, et al. HLA-B*5701 screening for hypersensitivity to abacavir. N Engl J Med. 2008; 358:568-579. [PubMed: 18256392]

59. Worm SW, Sabin C, Weber R, et al. Risk of myocardial infarction in patients with HIV infection exposed to specific individual antiretroviral drugs from the 3 major drug classes: the data collection on adverse events of anti-hiv drugs (D:A:D) study. J Infect Dis. 2010; 201:318-330. [PubMed: 20039804]

60. Calza L, Manfredi R, Colangeli V, et al. Efficacy and safety of atazanavir-ritonavir plus abacavirlamivudine or tenofovir-emtricitabine in patients with hyperlipidaemia switched from a stable protease inhibitor-based regimen including one thymidine analogue. AIDS Patient Care STDS. 2009; 23:691-697. [PubMed: 19739937]

61. Kumar PN, Rodriguez-French A, Thompson MA, et al. A prospective, 96-week study of the impact of Trizivir, Combivir/nelfinavir, and lamivudine/ stavudine/nelfinavir on lipids, metabolic parameters and efficacy in antiretroviral-naive patients: effect of sex and ethnicity. HIV Med. 2006; 7:85-98. [PubMed: 16420253] 
62. Shikuma CM, Yang Y, Glesby MJ, et al. Metabolic effects of protease inhibitor-sparing antiretroviral regimens given as initial treatment of HIV-1 Infection (AIDS Clinical Trials Group Study A5095). J Acquir Immune Defic Syndr. 2007; 44:540-550. [PubMed: 17245230]

63. Brothers $\mathrm{CH}$, Hernandez JE, Cutrell AG, et al. Risk of myocardial infarction and abacavir therapy: no increased risk across 52 GlaxoSmithKline-sponsored clinical trials in adult subjects. J Acquir Immune Defic Syndr. 2009; 51:20-28. [PubMed: 19282778]

64. Benson, CRH.; Zheng, E.; Koletar, S., et al. No association of abacavir use with risk of myocardial infarction or severe cardiovascular disease events: results from ACTG A5001; presented at: 16th Conference on Retroviruses and Opportunistic Infections; February 8-11, 2009; Montreal, Canada. Abstract Abstract \#721

65. Durand, MSO; Baril, JA. Relation between use of nucleoside reverse transcriptase inhibitors (NRTI) and risk of myocardial infarction (MI): a nested case control study using Quebec's public health insurance database (QPHID); presented at: 5th IAS Conference on HIV Pathogenesis, Treatment, and Prevention; July 19-22, 2009; Cape Town, South Africa. Abstract Abstract \#TUPEB175

66. Bedimo, RWA.; Dreschler, H.; Tebas, P. Abacavir use and risk of acute myocardial infarction and cerebro-vascular disease in the HAART era; presented at: 5th IAS Conference on HIV Pathogenesis, Treatment, and Prevention; July 19-22, 2009; Cape Town, South Africa. Abstract Abstract \#MOAB202

67. Post FA, Campbell LJ. Abacavir and increased risk of myocardial infarction. Lancet. 2008; 372:803. author reply 4-5. [PubMed: 18774412]

68. Palella, FGS.; Elion, R.; Benning, L., et al. Inflammatory markers among abacavir and nonabacavir recipients in the Womens' Interagency HIV study and the Multicenter AIDS cohort study; presented at: 16th Conference on Retroviruses and Opportunistic Infections; February 8-11, 2009; Montreal, Canada. Abstract Abstract \#150LB

69. Martinez, ELM.; Perez, I. No evidence for recent abacavir/lamivudine use in promoting inflammation, endothelial dysfunction, hypercoaguability, or insulin resistance in virologically suppressed HIV-infected patients: a substudy of the BICOMBO randomized clinical trial; 5th IAS Conference on HIV Pathogenesis, Treatment, and Prevention; July 19-22, 2009; Cape Town, South Africa. Abstract \#MOAB203

70. Hsue PY, Hunt PW, Wu Y, et al. Association of abacavir and impaired endothelial function in treated and suppressed HIV-infected patients. AIDS. 2009; 23:2021-2027. [PubMed: 19542863]

71. Satchell, COCE.; Peace, A.; Cotter, A.; Sheehan, G.; Tedesco, T.; Doran, P.; Powderly, W.; Kenny, D.; Mallon, P. Platelet hyper-reactivity in HIV-1 infected patients on abacavir-containing ART; presented at: 15th Conference on Retroviruses and Opportunistic Infections; July 19-22, 2009; Montreal, Canada. Abstract Abstract \#151LB

72. DeJesus E, Herrera G, Teofilo E, et al. Abacavir versus zidovudine combined with lamivudine and efavirenz, for the treatment of antiretroviral-naive HIV-infected adults. Clin Infect Dis. 2004; 39:1038-1046. [PubMed: 15472858]

73. Lamarca A, Clumeck N, Plettenberg A, et al. Efficacy and safety of a once-daily fixed-dose combination of abacavir/lamivudine compared with abacavir twice daily and lamivudine once daily as separate entities in antiretroviral-experienced HIV-1-infected patients (CAL30001 Study). J Acquir Immune Defic Syndr. 2006; 41:598-606. [PubMed: 16652033] 


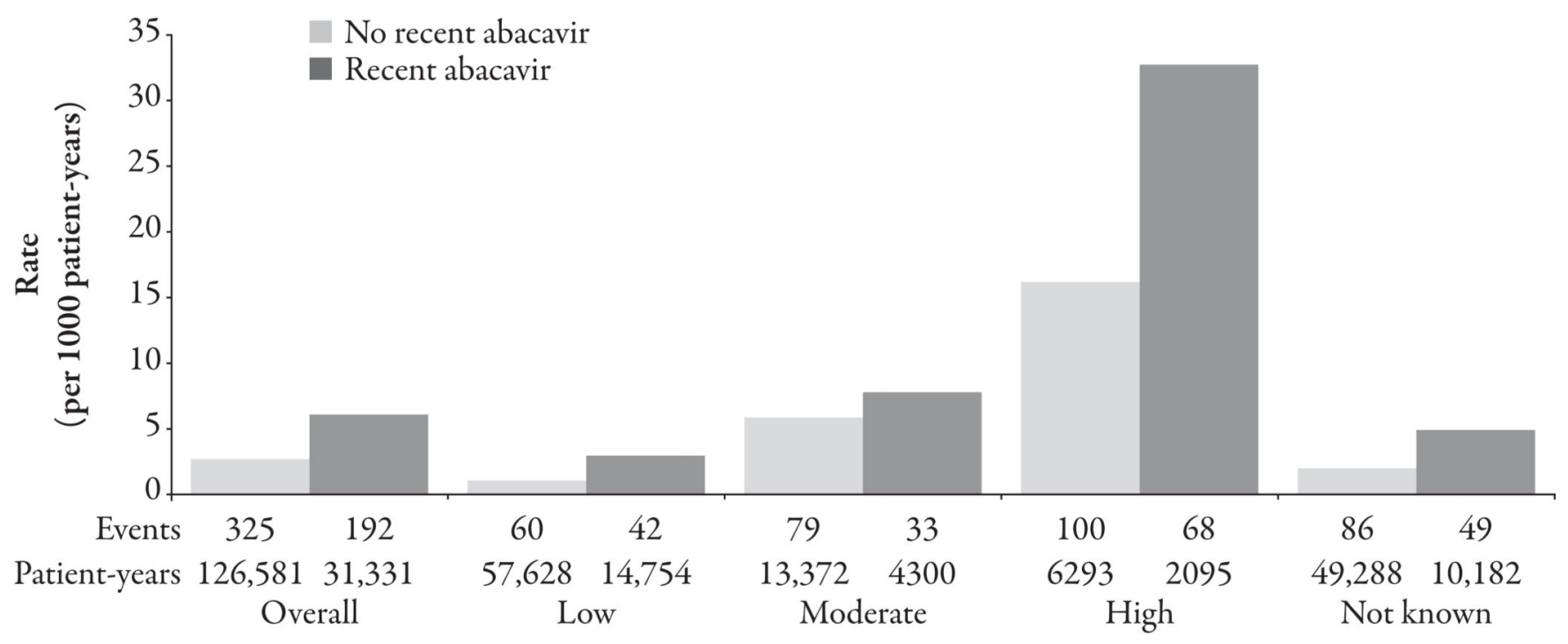

Predicted 10-year risk of coronary heart disease

Figure 1.

From the Data Collection on Adverse Events of Anti-HIV Drugs (D:A:D) study. ${ }^{10}$ Rates of myocardial infarction, stratified by predicted 10-year risk of coronary heart disease and use of abacavir. Reprinted from The Lancet, Vol. 371, Sabin CA, et al. Use of nucleoside reverse transcriptase inhibitors and risk of myocardial infarction in HIV-infected patients enrolled in the D:A:D study: a multi-cohort collaboration. Pages 1417-1426. Copyright (C) 2008, with permission from Elsevier 


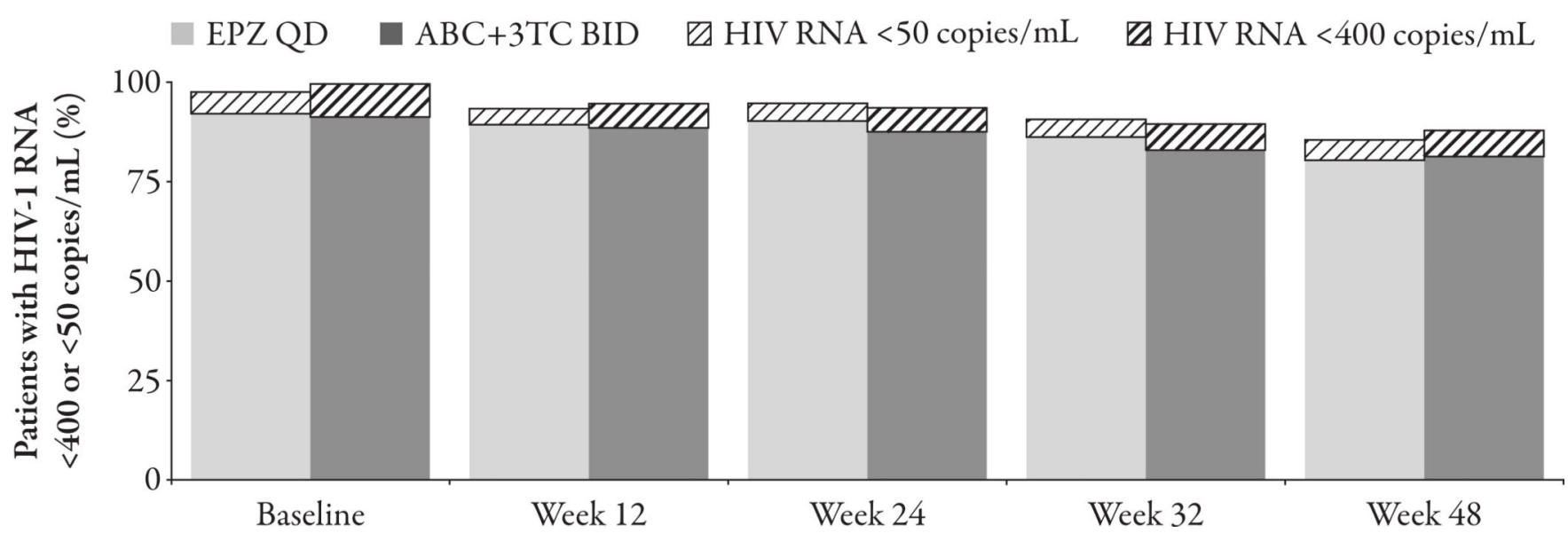

Figure 2.

From the ESS30008 (SEAL) study. ${ }^{54}$ Persistent virologic suppression with either once-daily fixed-dose abacavir and lamivudine (Epzicom) or twice-daily abacavir and lamivudine. $\mathrm{ABC}+3 \mathrm{TC} \mathrm{BID}=$ fixed-dose combination abacavir/lamivudine twice daily; EPZ $\mathrm{QD}=$ Epzicom once daily. Reproduced with permission from: Sosa N, et al. Abacavir and lamivudine fixed-dose combination tablet once daily compared with abacavir and lamivudine twice daily in HIV-infected patients over 48 weeks (ESS30008, SEAL). J Acquir Immune Defic Syndr. 2005;40:422-427. 

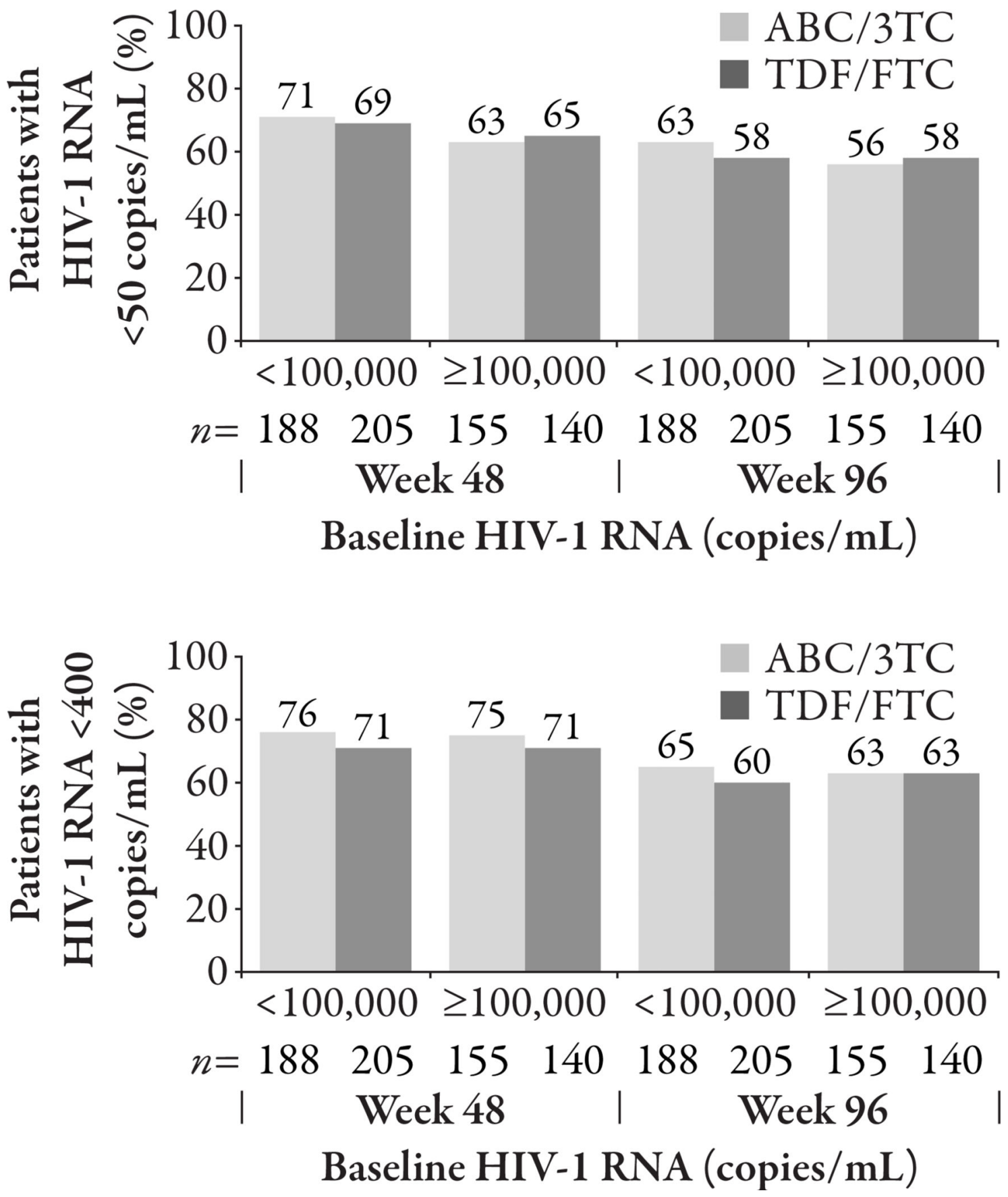

Figure 3.

From the HIV Study with Epzicom And Truvada (HEAT) trial. ${ }^{53}$ Proportion of patients with HIV viral load $<50$ and $<400$ copies/mL stratified by baseline viral load. ABC/ 3TC=Epzicom; TDF/FTC=Truvada. Reproduced with permission from: Smith KY, et al. Randomized, double-blind, placebo-matched, multicenter trial of abacavir/lamivudine or tenofovir/emtricitabine with lopinavir/ritonavir for initial HIV treatment. AIDS. 2009;23:1547-1556. 

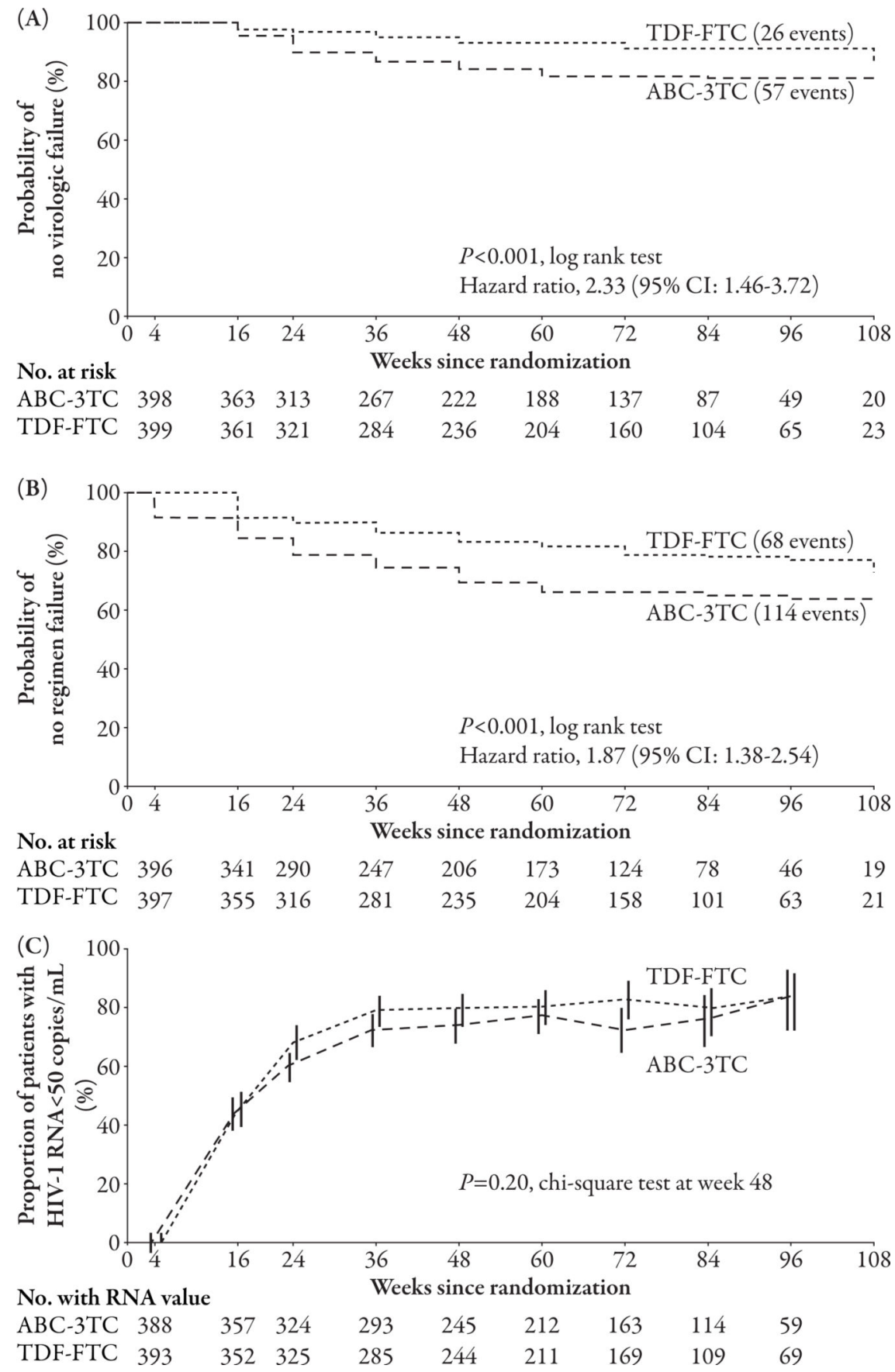

Figure 4.

From the ACTG 5202 study. ${ }^{14}$ (A) Time to virologic failure; (B) First occurrence of either virologic failure or modification of NRTI; (C) Proportion of patients with HIV viral load $<50$ copies $/ \mathrm{mL}$. All three of these results are restricted to patients with baseline HIV viral load level $\geq 100,000$ copies $/ \mathrm{mL}$. ABC-3TC=Epzicom; TDF-FTC=Truvada. Reproduced with permission from: Sax PE, et al. Abacavir-lamivudine versus tenofovir-emtricitabine for 
initial HIV-1 therapy. N Engl J Med. 2009;361:2230-2240. Copyright (c) 2009 Massachusetts Medical Society. All rights reserved. 